\title{
Identification of the role of TRPM8 in glioblastoma and its effect on proliferation, apoptosis and invasion of the U251 human glioblastoma cell line
}

\author{
JIANPING ZENG $^{1 *}$, YE WU $^{1 *}$, SIYI ZHUANG ${ }^{2}$, LIPING QIN $^{3}$, SHUSHAN HUA $^{2}$, \\ RAJNEESH MUNGUR ${ }^{1}$, JIANWEI PAN ${ }^{1}$, YU ZHU ${ }^{1}$ and RENYA ZHAN ${ }^{1}$
}

\author{
${ }^{1}$ Department of Neurosurgery, The First Affiliated Hospital of Medical School, Zhejiang University, \\ Hangzhou, Zhejiang 310003; ${ }^{2}$ Department of Neurobiology, Zhejiang University School of Medicine; \\ ${ }^{3}$ Institute of Aging Research, Hangzhou Normal University, Hangzhou, Zhejiang 310012, P.R. China
}

Received December 3, 2018; Accepted July 12, 2019

DOI: 10.3892/or.2019.7260

\begin{abstract}
Glioblastoma multiforme (GBM) is the most commonly occurring brain cancer, and is characterized by its poor patient outcomes. The present study examined the mRNA expression levels of the transient receptor potential melastatin (TRPM) family in various types of cancer using the ONCOMINE database, along with their corresponding expression profiles in an array of cancer cell lines based on the Cancer Cell Line Encyclopedia (CCLE) datasets. Kaplan-Meier plotter survival analysis via the Chinese Glioma Genome Atlas (CGGA) database was also used to evaluate the prognostic value of transient receptor potential melastatin 8 (TRPM8). For the activity test on the TRPM8 channel, patch-clamp recordings and $\mathrm{Ca}^{2+}$ measurements by fluorescence imaging of Fluo-4am were performed. Short hairpin RNA (shRNA) targeting TRPM8 was designed, synthesized and then transfected into the U251 cells via Lipofectamine 2000. The expression of extracellular singnal-regulated kinase (ERK), cyclin D1 and Bcl-2 were detected by performing western blotting and immunofluorescence. The apoptosis, proliferation and invasion of glioma cells were detected by using flow cytometry, and CCK- 8 and Transwell invasion assays. In the present study, TRPM8 was distinctively upregulated in GBM cell lines. TRPM8 is functional and has the characteristic of outward rectification, which was verified via electrophysiology and $\mathrm{Ca}^{2+}$ fluorescence imaging in
\end{abstract}

Correspondence to: Professor Renya Zhan, Department of Neurosurgery, The First Affiliated Hospital of Medical School, Zhejiang University, 79 Qingchun Road, Hangzhou, Zhejiang 310003, P.R. China

E-mail: 1196057@zju.edu.cn

*Contributed equally

Key words: transient receptor potential melastatin 8, glioblastoma multiforme, bioinformatics, function, U251
U251 cells. The western blot and immunofluorescence results revealed that the expression of ERK, cyclin D1 and Bcl-2 were decreased in the shRNA interference group. The CCK- 8 assay demonstrated that the proliferation ability of U251 cells in the U251/TRPM8 group was higher than that in the U251 group and $\mathrm{U} 251 / \mathrm{Con}$ group $(\mathrm{P}<0.05)$. The result of the Transwell invasion assay indicated that the invasion of human glioblastoma U251 cells was positively correlated with the expression level of TRPM8. Collectively, the results of the present study indicated that $\mathrm{Ca}^{2+}$-permeable TRPM8 nonselective cation channels contribute to survival, proliferation, apoptosis, and local tumor invasion of glioblastoma. Therefore, TRPM8 is a promising biomarker for aggressiveness of GBM, and a potential target in future anti-glioblastoma therapies.

\section{Introduction}

Glioblastoma multiforme (GBM) is a highly malignant primary intracranial tumor with an average survival time after diagnosis of only 12-14 months, and a 5-year survival rate of merely $9 \%(1,2)$. At present, the standard treatment for GBM is surgical resection followed by radiotherapy combined with concurrent and/or adjuvant temozolomide (TMZ) chemotherapy $(3,4)$. While there have been numerous studies on immunotherapy and gene therapy for GBM, the effects have not been completely verified due to inconsistencies in the treatment methods and evaluation criteria. These, in turn, have highlighted the need for other effective therapies, such as biological therapy, combination therapy, interstitial brachytherapy, minimally invasive technical and ion-channel targeted therapy. Members of the transient receptor potential (TRP) cation channel superfamily have been observed to perform a myriad of functions, including temperature perception, pain transduction, vasorelaxation, male fertility and tumorigenesis (5-7). This emerging evidence has strongly supported the hypothesis that transient receptor potential melastatin 8 (TRPM8), which also plays a prominent role in thermoregulation, is one of the most promising novel therapeutic targets in cancer treatment (8-10). However, the functions of TRPM8 in contribution to tumorigenesis in GBM and the precise 
mechanisms of TRPM8 function in glioma cells have yet to be completely elucidated. The present study initially evaluated TRPM8 as a promising biomarker in GBM using public database analysis. Experiments were subsequently performed to verify whether TRPM8 serves an important role in the apoptosis, proliferation and invasion of human glioblastoma U251 cells.

\section{Materials and methods}

ONCOMINE analysis. ONCOMINE (http://www.oncomine. org), is an online cancer microarray database, and was used in the present study to analyze the transcription levels of the TRPM family in different types of human cancers. The mRNA expression of the TRPM family in clinical cancer specimens were compared with that in normal controls. The present study set the thresholds as follows: The gene rank, P-value and fold change were defined as $10 \%, 0.01$ and 2 , respectively.

Cancer Cell Line Encyclopedia (CCLE) analysis. The CCLE (https://portals.broadinstitute.org/ccle/home) provides public access to genomic data, analysis and visualization for $\sim 1,000$ cell lines. 'TRPM8' was searched in the CCLE database and the expression data of TRPM8 from the available cancer cell lines in a series of cancers was verified by using cell line data from CCLE.

Kaplan-Meier plotter survival analysis. The survival data were downloaded from CGGA (http://www.cgga.org.cn), a newly developed interactive web server for analyzing the gene expression data of 325 patients (203 males and 122 females). The samples were categorized into two groups (high and low TRPM8 expression levels) using the median gene expression value and were compared using a Kaplan-Meier survival plot. The investigators established overall survival (OS) time as the prognostic value of patients with glioblastoma. Survival analysis with $\mathrm{P}<0.05$ was considered to indicate a statistically significant difference.

Cell culture and cell transfection. The human glioblastoma U251 cells were kindly gifted by G.F. Vande Woude, (Van Andel Research Institute, Grand Rapids, MI, USA). The human glioblastoma U251 cells were cultured in DMEM containing $10 \%$ fetal bovine serum and placed in a humidified cell incubator with $5 \% \mathrm{CO}_{2}$ at $37^{\circ} \mathrm{C}$.

Cell monolayers (at $70 \%$ confluency) were transfected with the pEGFP-C1-TRPM8 plasmids and the enhanced green fluorescent protein plasmid-C1 (pEGFP-C1) vector using Lipofectamine 2000 (Thermo Fisher Scientific, Inc.) according to the manufacturer's protocol. shRNA targeting TRPM8 was designed and inserted into the pGPU6/GFP/Neo vector (Thermo Fisher Scientific, Inc.). shRNA-TRPM8 was also transfected into human glioblastoma U251 cells using Lipofectamine 2000 (Thermo Fisher Scientific, Inc.), according to the manufacturer's protocol.

Reverse transcription-quantitative polymerase chain reaction $(R T-q P C R)$. Total RNA was extracted from the cells using TRIzol reagent (Invitrogen; Thermo Fisher Scientific, Inc.) according to the manufacturer's protocol.
The cDNA Synthesis kit (Takara Biotechnology Co., Ltd.) was used for the synthesis of cDNA according to the manufacturer's protocol. RT-qPCR was performed using The PrimeScript $^{\mathrm{TM}}$ II Reverse Transcriptase kit obtained from Takara Biotechnology Co., Ltd. The $2^{-\Delta \Delta \mathrm{Cq}}$ method was used to calculate the expression level (defined as the fold change) of TRPM8 compared with GAPDH expression. The primer sequences were as follows: TRPM8 sense chain, 5'-TATCTT ACTGAACACCTGTAGTCCCAG-3' and antisense chain, 5'-TGAGTTTAGTGTATTCAAAGCTGAGAAA-3' (256 bp); GAPDH sense chain, 5'-AGTGAAGTCGTCGTCAAC-3' and antisense chain, 5'-CGCTCCTGAGATGTGAT-3' (32 bp). The experiment was repeated 3 times.

Western blot assay. Cells were lysed in RIPA buffer (KeyGen Biotech. Co., Ltd., Nanjing, China) and protein in supernatant extracts was quantified using a BCA Protein Assay kit (Beyotime Institute of Biotechnology). A total of $50 \mu \mathrm{g}$ per lane of total cell lysates was resolved on $12 \%$ sodium dodecyl sulfate-polyacrylamide gel electrophoresis (SDS-PAGE) gels and transferred onto polyvinylidene fluoride (PVDF) membranes (EMD Millipore). The blots were incubated with a primary antibody overnight at $4^{\circ} \mathrm{C}$. The blots were subsequently incubated with horseradish peroxidase-linked secondary anti-rabbit or anti-mouse antibody (dilution 1:3,000; cat. nos. STAR71D800GA and STAR117D800GA; Bio-Rad Laboratories, Inc.). Immunoreactivity was detected using enhanced chemiluminescence (Amersham; GE Healthcare). Densitometric analysis was performed using Quantity One software (Bio-Rad Laboratories, Inc.). GAPDH was used as a loading control. TRPM8 (dilution 1:1,000; cat. no. sc-169688), ERK (dilution 1:1,000; cat. no. sc-271270), cyclin D1 (dilution 1:1,000; cat. no. sc-8396), Bcl-2 (dilution 1:1,000; cat. no. sc-70411), and GAPDH (dilution 1:1,000; cat. no. sc-51907) antibodies were purchased from Santa Cruz Biotechnology, Inc.

Electrophysiology and $\mathrm{Ca}^{2+}$ measurements by fluorescence imaging of Fluo-4am. For the activity test on the TRPM8 channel, patch-clamp recordings were performed in whole-cell configuration at room temperature using Axonpatch 200B (Molecular Devices, LLC) or HEKA EPC10 (HEKA Elektronik GmbH) amplifier. U251 cells were seeded on coverslips prior to recording the TRPM8 currents via electrophysiology with the standard extracellular solution (ECS) containing (in $\mathrm{mM}$ ): $130 \mathrm{NaCl}, 5 \mathrm{KCl}$, 10 D-glucose, 10 HEPES, $1.2 \mathrm{MgCl}_{2}$ and $1.5 \mathrm{CaCl}_{2}, \mathrm{pH} 7.4$. For specificity evaluation of characteristics of the TRPM8 channel of U251 cells, menthol $(100 \mu \mathrm{M})$ was added in ECS to activate TRPM8, and AMTB Hydrate $(50 \mu \mathrm{M})$ was applied in ECS to inhibit TRPM8. $\mathrm{Ca}^{2+}$ measurements by fluorescence imaging of Fluo-4am were subsequently performed for further evaluation. U251 cells grown on $10-\mathrm{mm}$ glass coverslips were incubated with Fluo- 4 am for $0.5 \mathrm{~h}$ in the dark at $37^{\circ} \mathrm{C}$ for the $\mathrm{Ca}^{2+}$ measurements. The cells were then washed with HBSS containing calcium and placed on a confocal microscope. The intracellular calcium images were recorded using Evolve 512 EMCCD (Teledyne Photometrics) and menthol using RSC-200 Rapid Solution Changer (Bio-Logic Science Instruments). Then the fluorescence intensities were analyzed using ImageJ 
software (version 1.8.0; National Institutes of Health). Data were presented as the traces of average fluorescence intensities values.

Cell proliferation assay. Cell viability was measured using a Cell Counting Kit-8 (CCK-8) assay (Dojindo Molecular Laboratories, Inc.). Transfection of TRPM8 or the non-specific control was performed in 96-well plates in quadruplicate. Cell culture medium was replaced at $24 \mathrm{~h}$ following transfection. CCK-8 $(10 \mu \mathrm{l})$ was added to each well, which also contained $100 \mu \mathrm{l}$ medium. Following a 2 -h incubation with the CCK-8 solution, the absorbance at $450 \mathrm{~nm}$ was measured at $48 \mathrm{~h}$ following transfection. Each experiment was performed in triplicate.

Apoptosis assay. Apoptosis was determined using the Annexin V-PE/7AAD apoptosis kit (Nanjing KeyGen Biotech Co., Ltd.) according to the manufacturer's protocol. Briefly, after $72 \mathrm{~h}$ of culture, the cells were collected and washed twice in PBS and re-suspended at a density of $1 \times 10^{3}$ cells $/ \mathrm{ml}$. The transfected cells were subsequently stained with Annexin V and propidium iodide in the dark and 7-ADD for $20 \mathrm{~min}$ and analyzed using a FACSCalibur instrument.

Immunofluorescence. The cells were seeded on coverslips and incubated for $24 \mathrm{~h}$ under normoxic conditions. Subsequently, the cells were fixed with $4 \%$ paraformaldehyde at room temperature and permeabilized with $0.2 \%$ Triton $\mathrm{X}-100$ for $10 \mathrm{~min}$. The cells were subsequently washed with PBS and blocked in PBS containing 5\% bovine serum albumin (Sigma-Aldrich; Merck KGaA) for $90 \mathrm{~min}$. The cells were washed with PBS and the primary antibodies were diluted with PBS containing 2\% BSA as follows: Rabbit anti-phospho-specific ERK (cat. no. sc-271270), Bcl-2 (cat. no. sc-70411), phospho-specific c-Jun N-terminal protein kinase (JN; cat. no. sc-7345), caspase-3 (cat. no. sc-271759), phospho-specific p38 mitogen-activated protein kinase (MAPK) (cat. no. sc-271759) antibodies (dilution 1:200; Santa Cruz Biotechnology, Inc.) and incubated overnight at $4^{\circ} \mathrm{C}$. The cells were then washed with PBS and incubated for $2 \mathrm{~h}$ with an anti-mouse fluorescent secondary antibody (cat. no. A10036; Invitrogen; Thermo Fisher Scientific, Inc.) at room temperature. Finally, DAPI (Beijing ComWin Biotech Co., Ltd.) was added to each sample for nuclear counterstaining. The coverglass was examined and photographed to reveal representative cells using an Olympus BX61WI-FV1200MPE confocal microscope (Olympus Corp.).

Transwell invasion assay. Transwell invasion was performed with 24-well Matrigel-coated chambers ( $8-\mu \mathrm{M}$ pore size) from BD Biosciences. Briefly, cells were permitted to grow to $\sim 75-80 \%$ confluence and were starved for $24 \mathrm{~h}$ by serum. Approximately $1 \times 10^{5}$ cells resuspended in $200 \mu 1$ serum-free medium were plated in the upper Transwell chamber. Then, $600 \mu \mathrm{l}$ of medium with $10 \%$ FBS was added to the bottom wells of the chambers. At $48 \mathrm{~h}$ following incubation, the Transwell insert was washed twice with PBS and fixed with $5 \%$ glutaraldehyde for $5 \mathrm{~min}$. The invading cells on the bottom surface of the membrane were stained for $20 \mathrm{~min}$ with $0.1 \%$ crystal violet. Then the membranes were observed at an x100 magnification using a light microscope (Olympus Corp.). The membrane was then dissolved by $10 \%$ acetic acid, and a multifunctional microplate reader was employed to measure the optical density (OD) value of each well at $570 \mathrm{~nm}$. The invasion rate $=$ OD 570 post-crystal violet staining/OD 490 at the time of inoculation. Each experiment was performed in triplicate.

Statistical analysis. All data in the present study are presented as the mean \pm standard deviation of at least three independent experiments. Statistical tests were performed using SPSS version 19.0.0 software (IBM Corp.). A two-tailed Student's t-test was used for comparisons between two groups. One-way analysis of variance (ANOVA) test and Bonferroni post hoc test were used for evaluating differences among multiple groups. $\mathrm{P}<0.05$ was considered to indicate a statistically significant difference.

\section{Results}

Compared with other TRPM family members, TRPM8 is markedly overexpressed in cancer of the brain and central nervous system (CNS). To assess the differences in mRNA expression of TRPM family members in human cancers and normal tissues in a variety of cancer types, the present study performed an analysis using the ONCOMINE database (Fig. 1). The results of the ONCOMINE analysis indicated that TPRM8 was elevated in cancer of the brain and CNS when compared with the normal tissue groups.

Corresponding expression profiles of TRPM8 in an array of cancer cell lines. By searching the CCLE (https://portals. broadinstitute.org/ccle/home), the results revealed that TRPM8 was highly expressed in all GBM cell lines (Fig. 2). These results indicated that TRPM8 may serve a unique role in the development of GBM, which was consistent with the results of the ONCOMINE analysis.

Kaplan-Meier plotter survival analysis of TRPM8 in patients with GBM. To validate the influence of TRPM8 on the prognosis of glioma patients, an overall survival (OS) analysis was performed using the Kaplan-Meier method via CGGA datasets. The results indicated that the high expression of TRPM8 mRNA was associated with a shorter overall survival (OS) time in all patients with GBM (P<0.0001) (Fig. 3). The survival analysis indicated that over-expression of TRPM8 was associated with worse survival in patients with GBM, suggesting TRPM8 serves a tumor-promoting role in GBM.

Characteristics of the TRPM8 channel. Menthol is a specific agonist against TRPM8, and AMTB hydrate is a TRPM8 blocker (11). To evaluate the characteristics of the TRPM8 channel, the whole cell membrane current of U251 cells was investigated by whole-cell patch-clamp recordings. Fig. 4A and B illustrate the whole cell membrane current of U251 cells at voltage ramps from -80 to $80 \mathrm{mV}$. The current-time curve of the TRPM8 channel after treatment with two consecutive $100-\mu \mathrm{M}$ menthol activations ruled out the effects of switching the drug delivery tube (Fig. 4A). When the transmembrane voltage was positive, the outward current was 


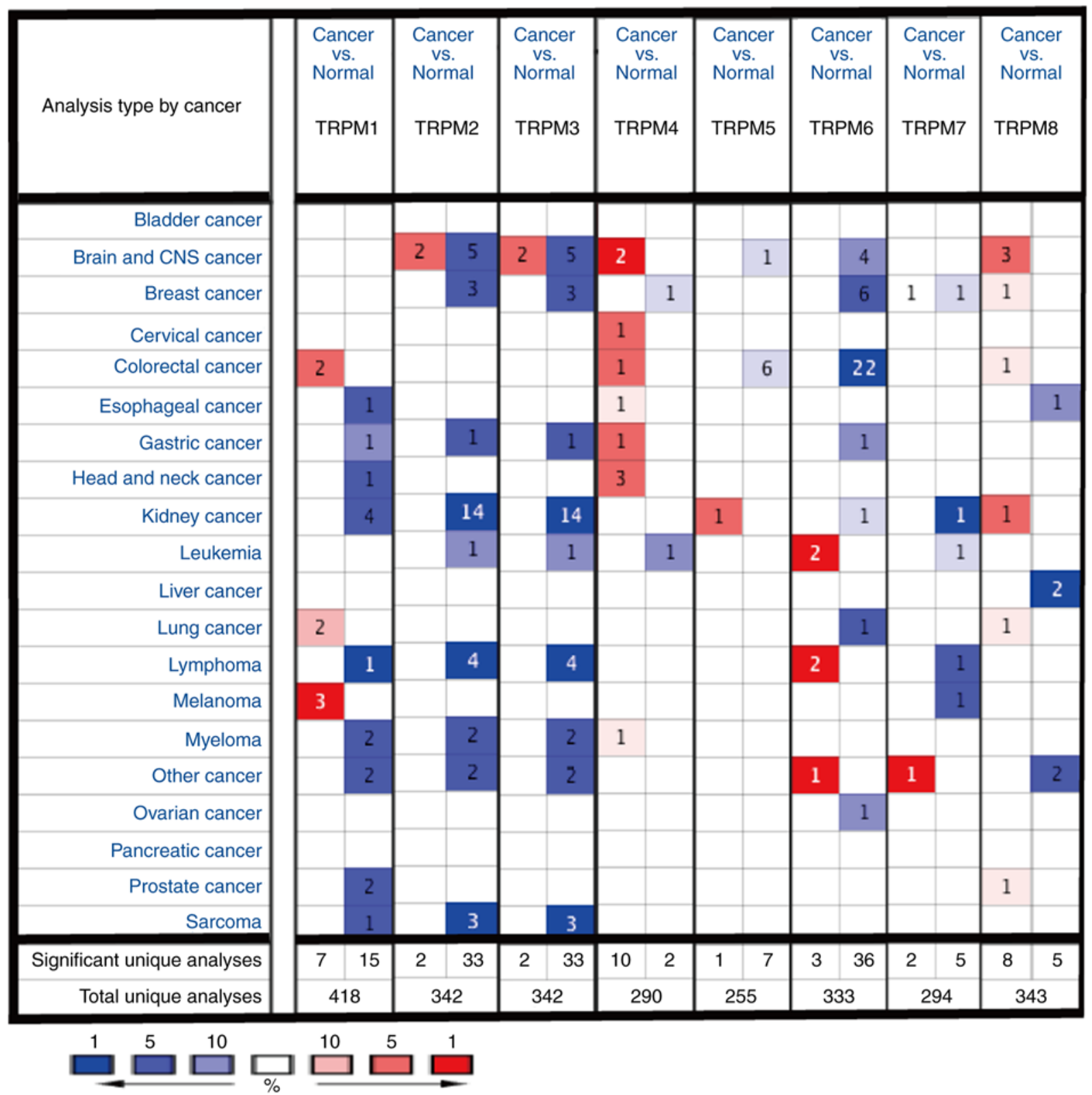

Figure 1. mRNA expression levels of TRPM protein family in human cancers. The number in the colored cell represents the number of analyses meeting thresholds. The color depth was determined by the gene rank. The more intense red (overexpression) or blue (underexpression) color indicates a highly significant overexpressed or underexpressed gene. TRMP8, transient receptor potential melastatin 8 .

markedly increased. The whole cell membrane currents were significantly increased when menthol $(100 \mu \mathrm{M})$ was added in ECS to activate TRPM8 (Fig. 4B), and was reversed by AMTB hydrate $(50 \mu \mathrm{M})$, further confirming that AMTB hydrate functions as a blocker of TRPM8. In addition, the fluorescence imaging results revealed that the ion channel activity of TRPM8 may be attributable to increased $\left[\mathrm{Ca}^{2+}\right]_{\mathrm{i}}$ following the addition of menthol. Menthol increased U251 $\left[\mathrm{Ca}^{2+}\right]_{\mathrm{i}}$, and this increase could be reversed following the removal of menthol (Fig. 4C). The ability of menthol to activate TRPM8 ion channels in the glioma cells was examined by overexpressing the channel in the U251 cells. The vehicle of pEGFP-C1 as control groups were also transfected into the $\mathrm{U} 251$ cells. The $\mathrm{Ca}^{2+}$ influx was monitored using Fluo-4am, and there was a slight increase following the overexpression of TRPM8. Following treatment with $100 \mu \mathrm{M}$ menthol, a significant increase in fluorescence was detected for TRPM8, whereas no fluorescence increase was observed for the control groups (Fig. 4D).

TRPM8 enhances the sensitivity of GBM cells to apoptosis. Firstly, by detecting the gene and protein expression levels of TRPM8 via RT-qPCR and western blot assay, the present study confirmed that transfection with shRNA-TRPM8 could significantly inhibit the mRNA and protein expression of TRPM8 ( $\mathrm{P}<0.01)$. Conversely, the overexpression of TRPM8 was significantly increased following transfection with the pEGFP-C1-TRPM8 plasmid $(\mathrm{P}<0.05)$. These results indicated that the downregulated and upregulated TRPM8 U251 cell lines were successfully constructed (named U251/shRNA and U251/TRPM8, respectively) (Fig. 5). As revealed in Fig. 6A, inactivation of TRPM8 expression could significantly increase the apoptosis rate $(\mathrm{P}<0.01)$, indicating that the modulation 

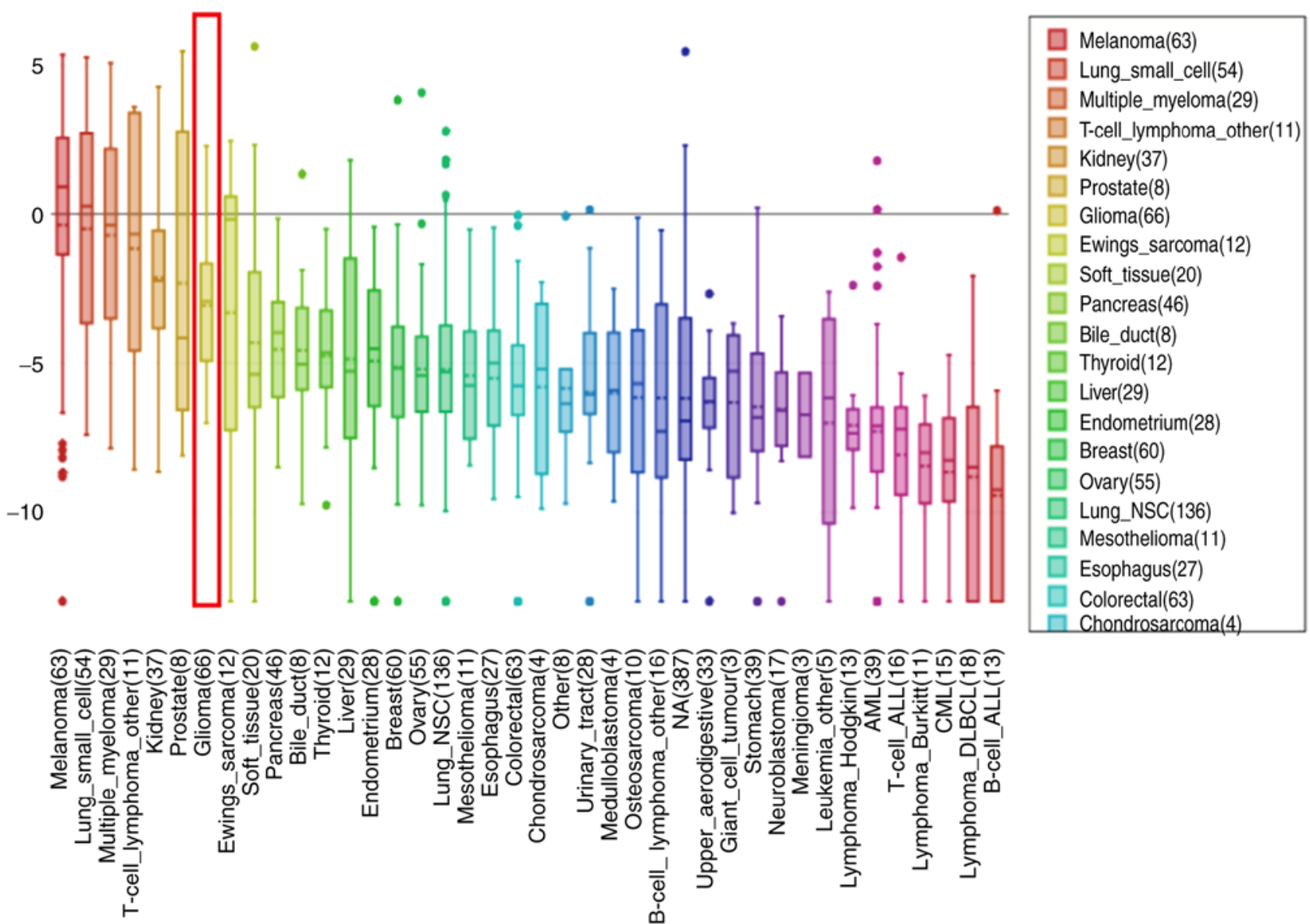

Figure 2. TRPM8 is distinctively overexpressed in GBM cell lines from the CCLE analysis. The mRNA expression level of TRPM8 ranked as the seventh highest in a variety of cancer cell lines (shown in the red frame). TRMP8, transient receptor potential melastatin 8; GBM, glioblastoma multiforme.

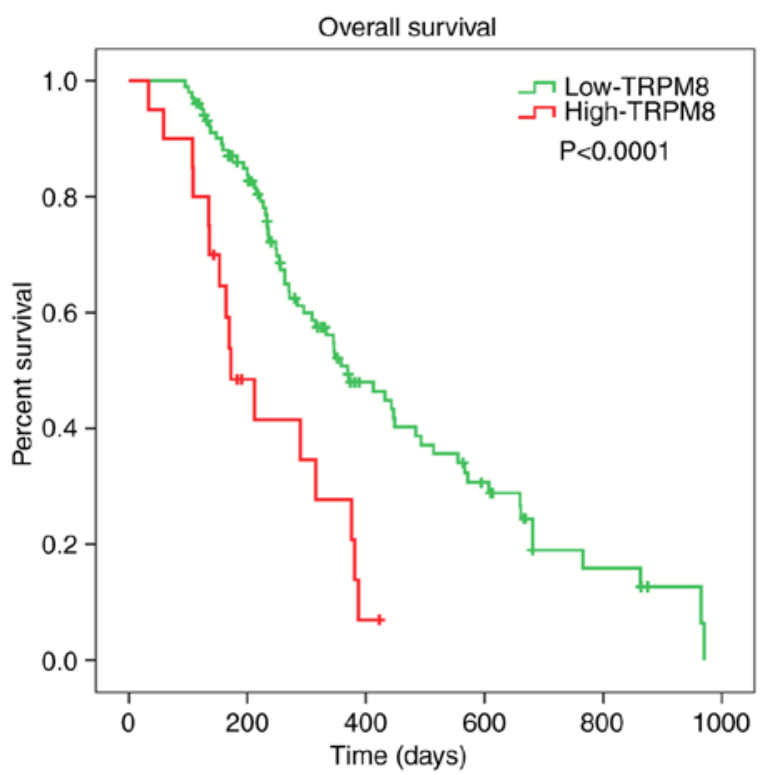

Figure 3. The prognostic values of TRPM8 in GBM according to the CGGA database. TRMP8, transient receptor potential melastatin 8 , glioblastoma multiforme; GBM, glioblastoma multiforme; CGGA, Chinese Glioma Genome Atlas.

of cell apoptosis was significantly affected by the expression of TRPM8. The expression levels of p-ERK, Bcl-2 were significantly decreased when TRPM8 was upregulated and increased when TRPM8 was downregulated in human glioblastoma U251 cells as revealed in Fig. 6B. The present study also confirmed these results via cellular immunofluorescence (Fig. 7). In addition, cellular immunofluorescence also revealed that the expression of phospho-specific JNK, caspase-3 and phospho-specific p38 MAPK were increased when TRPM8 was downregulated (Fig. 7).

TRPM8 regulates the proliferation and invasion abilities of human glioblastoma U251 cells. The results of the CCK-8 assay indicated that the proliferative ability of U251 cells in the U251/TRPM8 group was higher than that in the U251 and U251/Con groups $(\mathrm{P}<0.05)$; however, there was no significant difference between the U251 and U251/Con groups ( $\mathrm{P}>0.05)$. The details of the proliferation capacity are presented in Table I. In addition, the expression level of cyclin D1 was markedly altered when TRPM8 was regulated in human glioblastoma U251 cells (Fig. 6B). Collectively, the results demonstrated that the proliferation ability of U251 cells was significantly affected by the expression of TRPM8.

The invasion rate is a crucial cancer cell property that accurately reflects the invasive ability of human glioblastoma U251 cells. As illustrated in Fig. 8, the Transwell invasion assay revealed that the U251 cells in the U251/TRPM8 group had an increased number of invasive cells when compared with the U251 and U251/Con groups. According to the OD value, 

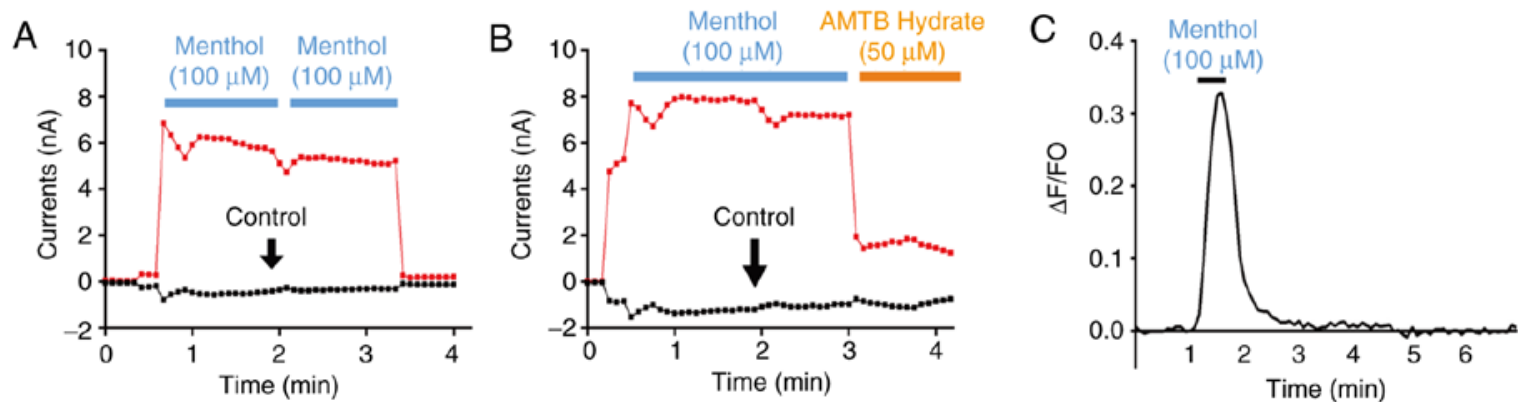

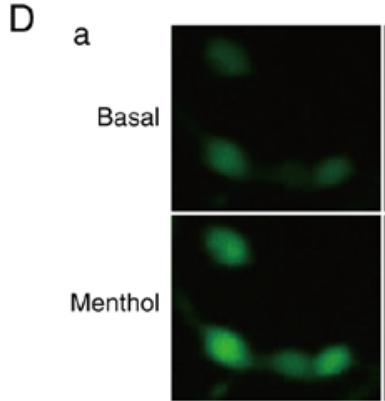

U251/TRPM8

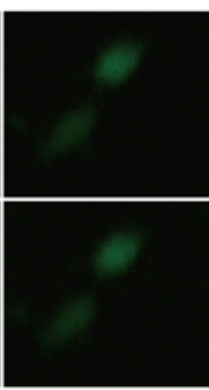

U251

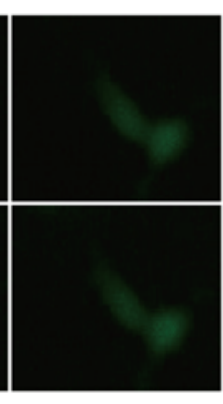

U251/Con

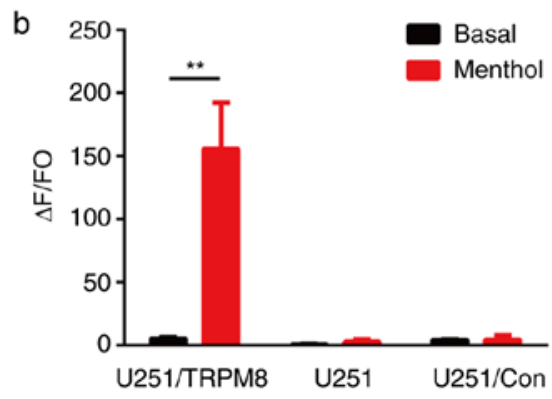

Figure 4. Whole-cell voltage clamp measurements and $\mathrm{Ca}^{2+}$ measurements by fluorescence imaging of Fluo-4am in human glioblastoma U251 cells. (A) The current-time curve of the TRPM8 channel after treating with two consecutive 100- $\mu \mathrm{M}$ menthol activations in the whole cell mode. (B) The effect of control with its agonist (100 $\mu \mathrm{M}$ menthol) and blocker (50 $\mu \mathrm{M}$ AMTB hydrate) on the TRPM8 channel. (C) Cells on glass coverslips were loaded with Fluo-4am and [Ca $\left.{ }^{2+}\right]$ were measured by inverted microscopy (AccuScope 3030). (D) In vitro activation of TRPM8 ion channels by menthol (100 uM). (a) Fluorescence images of glioma cells activated by menthol. (b) The fluorescence intensity of Fluo-4am activated by menthol. TRMP8, transient receptor potential melastatin 8 . ** $\mathrm{P}<0.01$.

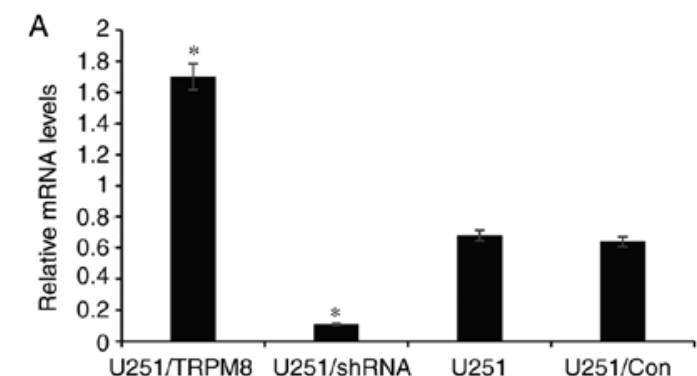

B

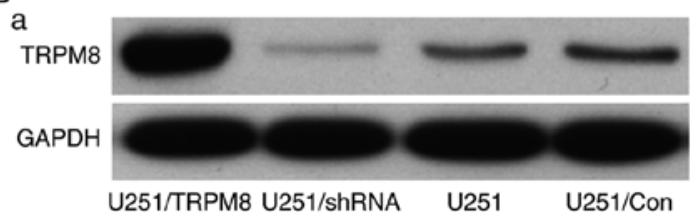

b

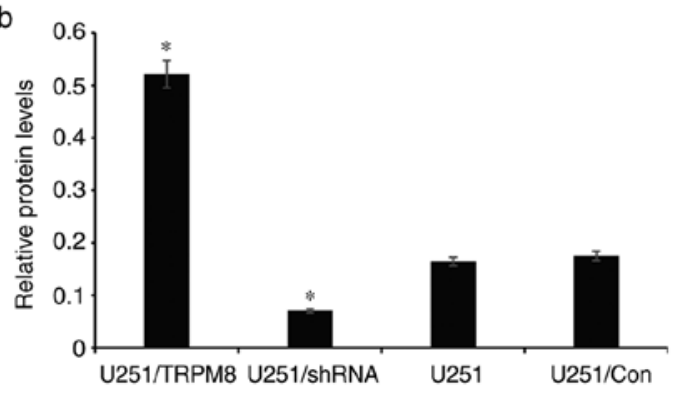

Figure 5. Relative TRPM8 expression at the gene and protein levels among different groups. (A) The relative gene expression level of TRPM8 in human glioblastoma U251 cells was significantly regulated when TRPM8 was knocked out or overexpressed as detected by RT-qPCR. (B-a and -b) The relative protein expression level of TRPM8 in human glioblastoma U251 cells was significantly upregulated following the overexpression of TRPM8 and downregulated with knockdown of TRPM8 as detected by western blotting. TRMP8, transient receptor potential melastatin $8 .{ }^{*} \mathrm{P}<0.05$.
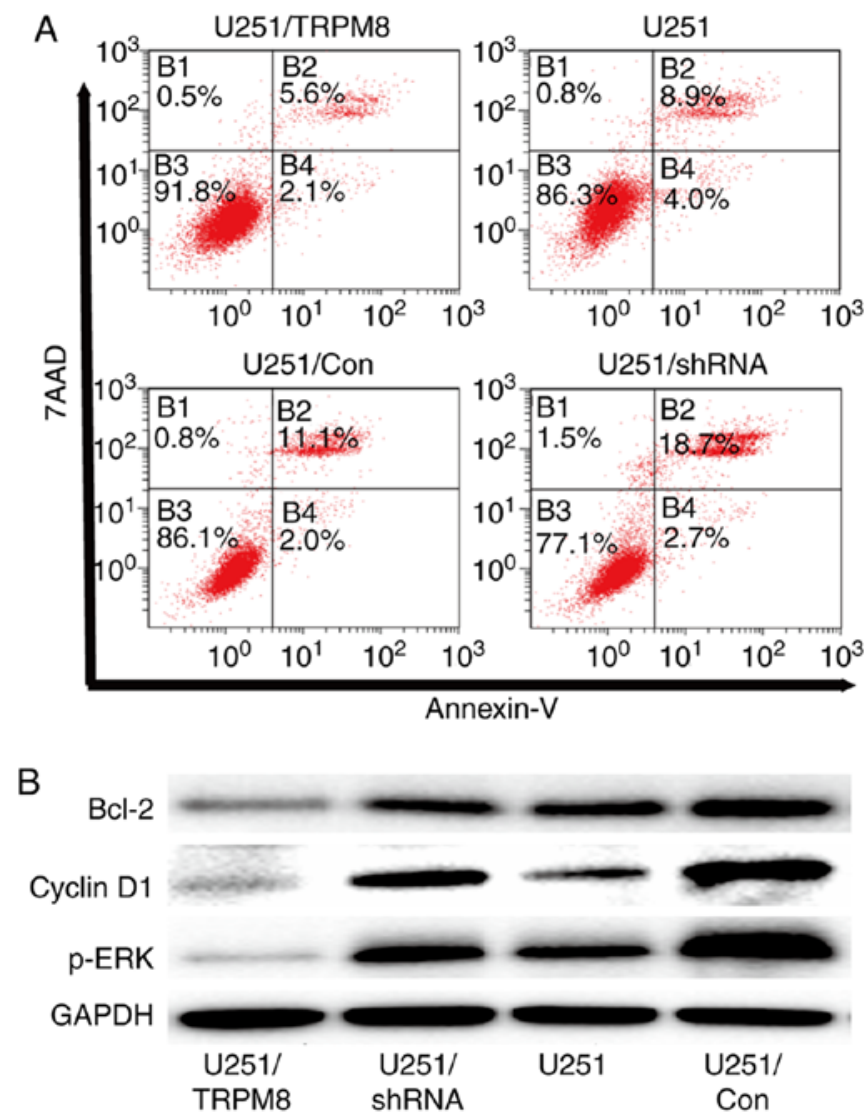

Figure 6. TRPM8 overexpression and TRPM8 inhibition markedly exhibit a decreased/increased apoptosis rate, respectively, in GBM cell lines. (A) The percent of apoptotic cells as determined by flow cytometry. (B) The expression of p-ERK, cyclin D1 and Bcl-2 in U251 cells when TRPM8 was upregulated or downregulated in human glioblastoma U251 cells. TRMP8, transient receptor potential melastatin 8 . 


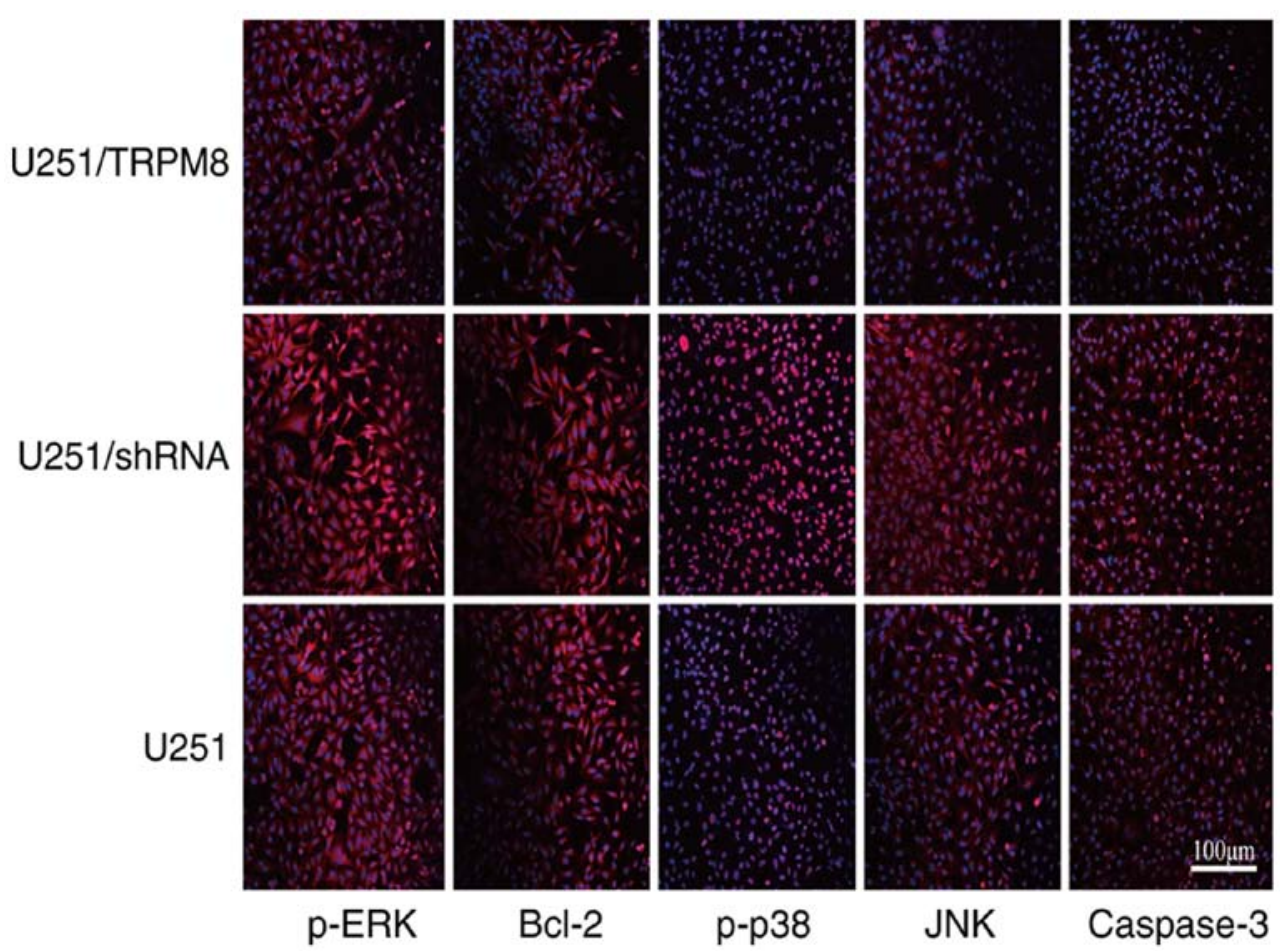

Figure 7. Immunofluorescence of phospho-specific ERK, Bcl-2, phospho-specific p38 MAPK, phospho-specific JNK, caspase-3 in human glioblastoma U251 cells. Cy3-immunofluorescence (red) indicates the proteins that were observed in the cells. DAPI (blue) indicates nuclear staining in cells. All images are merged (magnification, $\mathrm{x} 100)$.

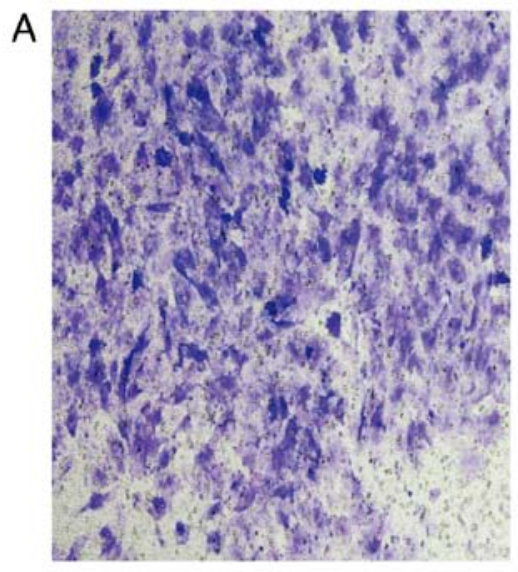

U251

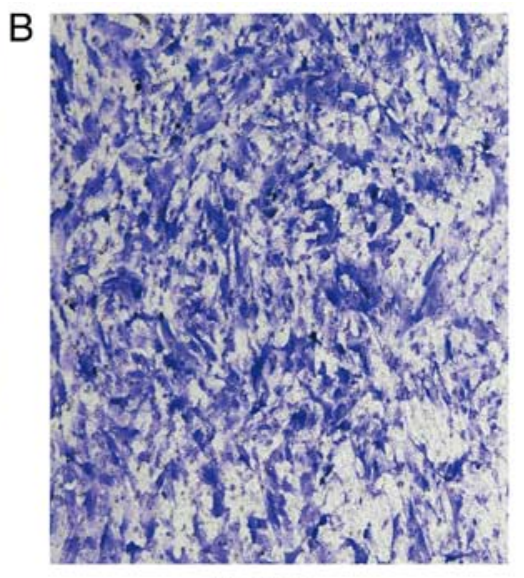

U251/Con

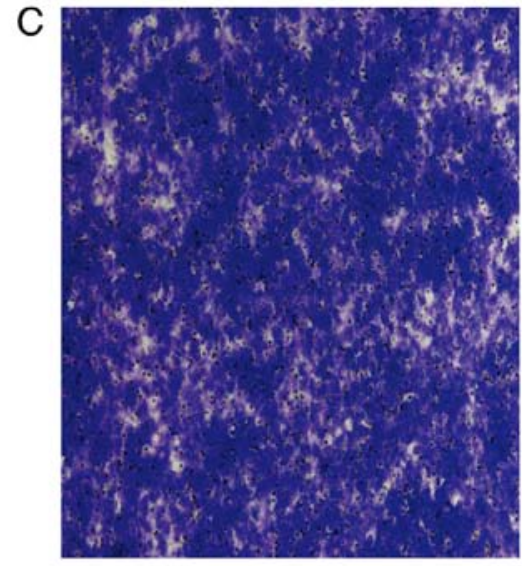

U251/TRPM8

Figure 8. Effect of TRPM8 on cell invasion. (A) Image of cells not transfected passing through Transwells. (B) Image of cells transfected with the pEGFP-C1-TRPM8 vector passing through the Transwell membrane. (C) Image of cell invasion that was increased in the cells with TRPM8 overexpression. TRMP8, transient receptor potential melastatin 8.

the transfer rate indicated that the U251/TRPM8 group had a higher invasion rate than the U251 and the U251/Con groups $(\mathrm{P}<0.05)$; however, the difference between the U251 and U251/Con groups was not statistically significant $(P>0.05)$ (Table I). Collectively, these results indicated that the invasion of human glioblastoma U251 cells was positively associated with the expression level of TRPM8.

\section{Discussion}

Glioblastoma is a Grade IV glioma, and is the most common and malignant tumors in glioma (12). Due to the
Table I. Comparison of the proliferation capacity (OD value) and invasion rate of human glioma U251 cells in each group $(\mathrm{x} \pm \mathrm{S})$.

\begin{tabular}{lcc}
\hline Groups & $\begin{array}{c}\text { Proliferation } \\
\text { capacity }\end{array}$ & $\begin{array}{c}\text { Invasion } \\
\text { rate }(\%)\end{array}$ \\
\hline U251 group & $0.147 \pm 0.012^{\mathrm{a}}$ & $1.363 \pm 0.035^{\mathrm{a}}$ \\
U251/Con group & $0.151 \pm 0.017^{\mathrm{a}}$ & $1.425 \pm 0.042^{\mathrm{a}}$ \\
U251/TRPM8 group & $0.361 \pm 0.024$ & $3.283 \pm 0.051$ \\
\hline
\end{tabular}

${ }^{\mathrm{a}} \mathrm{P}<0.05$, compared with the U251/TRPM8 group. 
limited efficacy of conventional treatments such as surgery, chemotherapy, and radiotherapy, there is an urgent need to identify novel and effective treatments. Over the past decade, numerous studies have made great progress in investigating the genetics and epigenetics of GBM $(13,14)$. Despite advances in early diagnosis and treatment, GBM has strong invasion cababilities and is characterized by its significantly shorter patient survival among intracranial tumors (15-18). There is a large volume of published studies describing the role of $\mathrm{Ca}^{2+}$ homeostasis (19-21). Multiple factors including $\mathrm{Ca}^{2+}$ signaling have been identified to influence the processes of tumorigenesis, proliferation and invasion (22). An increasing amount of evidence has recognized the importance of TRP cation channels, and the pivotal roles they serve in controlling these processes (23-25). TRP channels have been identified to exert pivotal functions in cellular calcium homeostasis and $\mathrm{Ca}^{2+}$ signaling (19). As the subclass of the TRP channels, TRPMs have varying permeability to $\mathrm{Ca}^{2+}$ and $\mathrm{Mg}^{2+}$ in various types of cancer $(5,26,27)$. Therefore, TRPM protein family members may serve attractive targets for anticancer therapeutic strategies or prognostic biomarkers to cure specific types of cancers $(26,28)$. It is common knowledge that TRPM8 is involved in the initiation and progression of tumors $(29,30)$. Although these findings undoubtledly require scrutinization, they suggested that TRPM8 may be involved in the transformation of normal cells to tumorigenic cells, which culminates in biological changes, such as tumor proliferation, apoptosis, gene transcription and angiogenesis (31). Morever, few studies have been performed where the gene expression data is correlated with the survival of patients $(8,9,11,28)$. Combined, TRPM8 may serve as an appropriate GBM biomarker, and this biomarker is urgently required for the early diagnosis and validation of interventions. The present study subsequently focused on describing the role of TRPM8 in GBM.

Survival analysis of TRPM8 in the present study demonstrated that overexpression of TRPM8 was significantly associated with OS of patients with GBM, which suggests that TRPM8 serves a tumor-promoting role in GBM. Furthermore, the results of the present study suggested that $\mathrm{Ca}^{2+}$-permeable TRPM8 nonselective cation channels were functional in human glioblastoma U251 cells. In addition, TRPM8 has the characteristic of outward rectification and may play an important role in regulating the biological behavior of glioblastoma cells. When menthol was used to activate the TRPM8 channel or overexpressed TRPM8, the $\left[\mathrm{Ca}^{2+}\right]_{i}$ was significantly increased.

The MAPK signaling pathway has been confirmed to be closely associated with the occurrence and development of tumors $(32,33)$. ERK, as an important regulator of the MAPK pathway, serves an important role in the proliferation and apoptosis of tumor cells $(33,34)$. ERK is an important subtribe of the family of mitogen-activated protein kinases (MAPKs). ERK can be activated by various growth factors and other mitogens (33). The activation of ERK is considered to be closely associated with cell proliferation, differentiation, migration, invasion, apoptosis and malignant transformation $(35,36)$. In addition, ERK has a direct regulatory effect on cyclin D1, which can accelerate cell mitosis and promote cell proliferation following activation; however, overexpression of ERK can activate nuclear factor- $\mathrm{\kappa B}(\mathrm{NF}-\mathrm{\kappa B})$, thereby inducing the expression of apoptosis-related proteins, such as Bcl-2 and Bcl-xL $(37,38)$. In addition, ERK can also exert anti-apoptotic effects by inhibiting the phosphorylation of pro-apoptotic proteins Bad and Bim (37). The present study revealed that the expression levels of ERK, Bcl-2 and cyclin D1 in human glioma cells were significantly lower than those in the U251/Con group when TRPM8 was downregulated. This indicated that part of the mechanism of TRPM8 in regulating the biological behaviors of human glioma cells may be via the regulation of ERK factors, thereby affecting the expression of apoptosis and proliferation-related factors. The activated caspase can hydrolyze important proteins including cell regulation, cell signal transduction and DNA repair, thus making the cells appear to wither (39). Apoptosis is characterized by specific morphological and biochemical features of death, in which caspase- 3 is the ultimate performer of apoptotic death (40). Is TRPM8-inhibiting tumor cell apoptosis also caspase-3 dependent? The results of the present study demonstrated that TRPM8 can inhibit the activity of JNK/MAPK signal transduction pathway. In addition, the results of the present study also suggested that part of the mechanism by which TRPM8 regulates the biological behavior of human glioma cells may be via the regulation of p38/MAPK signal transduction pathway, thereby affecting the expression level of apoptosis-related factors. However, it is worth noting that the regulation of the biological behavior of tumors involves multiple signaling pathways, and interactions between these signaling pathways are likely. In the future, a more in-depth study of the mechanism of action of TRPM8 will be conducted. It was hypothesized that the proliferation and apoptosis of human glioma cells regulated by TRPM8 may be associated with the MAPK pathway.

One of the most striking observations from data comparisons was the significant promotion of proliferative capacity of human glioblastoma U251 cells following the overexpression of TRPM8. Previous research revealed that the hepatocyte growth factor/scatter factor (HGF/SF) exhibited a modulatory effect on TRPM8 expression during oncogenesis $(8,41)$. In addition, the TPRM8 channel contributes to glioma invasion by inducing $\mathrm{Ca}^{2+}$ signaling, cytoskeleton changes and invasion (41). Activation of TRPM8 channel by its agonist, menthol, has been previously reported to stimulate the invasion of glioblastoma cells by increasing the intracellular $\mathrm{Ca}^{2+}$ concentration $(41,42)$, which is necessary for cell migration, and presumably tumor invasion $(8,41)$. Using human glioblastoma U251 cells, the present study investigated whether cell invasion and chemotaxis were dependent on TRPM8 channel activity, with the results indicating that TRPM8 significantly accelerated the invasion speed of U251 cells.

In summary, the present study indicated that the $\mathrm{Ca}^{2+}$-permeable channel of TRPM8 may have functional implications for glioma survival, proliferation, apoptosis and local tumor invasion. Therefore, TRPM8 may be utilized as an appealing anticancer target as well as a useful biomarker for cancer prognosis and treatment of GBM.

\section{Acknowledgements}

We sincerely express our thanks to Dr Wei Yang and Dr Yafei Yu for the advice and writing assistance on this experiment. At 
the same time, we appreciate Dr Tianfu Sun and Dr Kangli Xu for their help in analyzing and organizing the data.

\section{Funding}

The present study was supported by the Traditional Chinese Medicine Science and Technology Plan of Zhejiang province (grant no. 2019ZB030) and the Key Research \& Development (R\&D) Plan of Zhejiang Province (grant no. 2019C03095).

\section{Availability of data and materials}

The datasets used in this study are available from the corresponding author upon reasonable request.

\section{Authors' contributions}

JZ, YW and LQ performed the experiments and wrote the original draft. SZ performed the experiments. SH analyzed and interpreted the data. YZ was a guarantor and interpreted the data. JP conducted the bioinformatics analysis. RM polished the manuscript and was responsible for data curation. RZ conceived, designed, revised the study critically for important intellectual content. All authors read, reviewed and approved the manuscript and agree to be accountable for all aspects of the research in ensuring that the accuracy or integrity of any part of the work are appropriately investigated and resolved.

\section{Ethics approval and consent to participate}

Not applicable.

\section{Patient consent for publication}

Not applicable.

\section{Competing interests}

The authors declare that they have no competing interests.

\section{References}

1. Louis DN: Molecular pathology of malignant gliomas. Annu Rev Pathol 1: 97-117, 2006.

2. Jiang T, Mao Y, Ma W, Mao Q, You Y, Yang X, Jiang C, Kang C, Li X, Chen L, et al: CGCG clinical practice guidelines for the management of adult diffuse gliomas. Cancer Lett 375: 263-273, 2016.

3. Li MY, Yang P, Liu YW, Zhang CB, Wang KY, Wang YY, Yao K, Zhang W, Qiu XG, Li WB, et al: Low c-Met expression levels are prognostic for and predict the benefits of temozolomide chemotherapy in malignant gliomas. Sci Rep 6: 21141, 2016.

4. Stupp R, Dietrich PY, Ostermann Kraljevic S, Pica A, Maillard I, Maeder P, Meuli R, Janzer R, Pizzolato G, Miralbell R, et al: Promising survival for patients with newly diagnosed glioblastoma multiforme treated with concomitant radiation plus temozolomide followed by adjuvant temozolomide. J Clin Oncol 20: 1375-1382, 2002.

5. Chen J, Luan Y, Yu R, Zhang Z, Zhang J and Wang W: Transient receptor potential (TRP) channels, promising potential diagnostic and therapeutic tools for cancer. Biosci Trends 8: 1-10, 2014.

6. Wetsel WC: Sensing hot and cold with TRP channels. Int J Hyperthermia 27: 388-398, 2011.
7. Park YR, Chun JN, So I, Kim HJ, Baek S, Jeon JH and Shin SY: Data-driven analysis of TRP channels in cancer: Linking variation in gene expression to clinical significance. Cancer Genomics Proteomics 13: 83-90, 2016

8. Liu Z, Wu H, Wei Z, Wang X, Shen P, Wang S, Wang A, Chen W and $\mathrm{Lu} \mathrm{Y:} \mathrm{TRPM8:} \mathrm{A} \mathrm{potential} \mathrm{target} \mathrm{for} \mathrm{cancer} \mathrm{treatment.}$ J Cancer Res Clin Oncol 142: 1871-1881, 2016.

9. Alptekin M, Eroglu S, Tutar E, Sencan S, Geyik MA, Ulasli M, Demiryurek AT and Camci C: Gene expressions of TRP channels in glioblastoma multiforme and relation with survival. Tumour Biol 36: 9209-9213, 2015.

10. Zhang L and Barritt GJ: TRPM8 in prostate cancer cells: A potential diagnostic and prognostic marker with a secretory function? Endocr Relat Cancer 13: 27-38, 2006.

11. Burke RC, Bardet SM, Carr L, Romanenko S, Arnaud-Cormos D, Leveque P and O'Connor RP: Nanosecond pulsed electric fields depolarize transmembrane potential via voltage-gated $\mathrm{K}^{+}, \mathrm{Ca}^{2+}$ and TRPM8 channels in U87 glioblastoma cells. Biochim Biophys Acta Biomembr 1859: 2040-2050, 2017.

12. Yang M, Li Y, Chilukuri K, Brady OA, Boulos MI, Kappes JC and Galileo DS: L1 stimulation of human glioma cell motility correlates with FAK activation. J Neurooncol 105: 27-44, 2011.

13. Polivka J Jr, Polivka J, Holubec L, Kubikova T, Priban V, Hes O, Pivovarcikova $\mathrm{K}$ and Treskova I: Advances in experimental targeted therapy and immunotherapy for patients with glioblastoma multiforme. Anticancer Res 37: 21-33, 2017.

14. Stoyanov GS and Dzhenkov DL: On the concepts and history of glioblastoma Multiforme-Morphology, genetics and epigenetics. Folia Med (Plovdiv) 60: 48-66, 2018.

15. Jhaveri N, Chen TC and Hofman FM: Tumor vasculature and glioma stem cells: Contributions to glioma progression. Cancer Lett 380: 545-551, 2016.

16. Hardee ME and Zagzag D: Mechanisms of glioma-associated neovascularization. Am J Pathol 181: 1126-1141, 2012.

17. Stupp R, Mason WP, van den Bent MJ, Weller M, Fisher B, Taphoorn MJ, Belanger K, Brandes AA, Marosi C, Bogdahn U, et al: Radiotherapy plus concomitant and adjuvant temozolomide for glioblastoma. New Engl J Med 352: 987-996, 2005.

18. Sturm D, Bender S, Jones DT, Lichter P, Grill J, Becher O, Hawkins C, Majewski J, Jones C, Costello JF, et al: Paediatric and adult glioblastoma: Multiform (epi)genomic culprits emerge. Nat Rev Cancer 14: 92-107, 2014.

19. Roderick HL and Cook SJ: $\mathrm{Ca}^{2+}$ signalling checkpoints in cancer: Remodelling $\mathrm{Ca}^{2+}$ for cancer cell proliferation and survival. Nat Rev Cancer 8: 361-375, 2008.

20. Sun J, Mu H, Dai K and Yi L: Calreticulin: A potential anti-cancer therapeutic target. Pharmazie 72: 503-510, 2017.

21. Ohshima Y, Takata N, Suzuki-Karasaki M, Yoshida Y, Tokuhashi Y and Suzuki-Karasaki Y: Disrupting mitochondrial $\mathrm{Ca}^{2+}$ homeostasis causes tumor-selective TRAIL sensitization through mitochondrial network abnormalities. Int J Oncol 51: 1146-1158, 2017.

22. Rizzuto R, Pinton P, Ferrari D, Chami M, Szabadkai G, Magalhães PJ, Di Virgilio F and Pozzan T: Calcium and apoptosis: Facts and hypotheses. Oncogene 22: 8619-8627, 2003.

23. Fiorio Pla A and Gkika D: Emerging role of TRP channels in cell migration: From tumor vascularization to metastasis. Front Physiol 4: 311, 2013.

24. Hecquet CM, Zhang M, Mittal M, Vogel SM, Di A, Gao X, Bonini MG and Malik AB: Cooperative interaction of trp Melastatin channel transient receptor potential (TRPM2) with its splice variant TRPM2 Short variant is essential for endothelial cell apoptosis. Circ Res 114: 469-479, 2014.

25. Landsberg JW and Yuan JX: Calcium and TRP channels in pulmonary vascular smooth muscle cell proliferation. News Physiol Sci 19: 44-50, 2004.

26. Hantute-Ghesquier A, Haustrate A, Prevarskaya N and Lehen'kyi V: TRPM family channels in cancer. Pharmaceuticals (Basel) 11: pii: E58, 2018.

27. Gaunt HJ, Vasudev NS and Beech DJ: Transient receptor potential canonical 4 and 5 proteins as targets in cancer therapeutics. Eur Biophys J 45: 611-620, 2016.

28. Wong KK, Banham AH, Yaacob NS and Nur Husna SM: The oncogenic roles of TRPM ion channels in cancer. J Cell Physiol: Feb 2, 2019 doi: 10.1002/jcp.28168 (Epub ahead of print).

29. Okamoto Y, Ohkubo T, Ikebe T and Yamazaki J: Blockade of TRPM8 activity reduces the invasion potential of oral squamous carcinoma cell lines. Int J Oncol 40: 1431-1440, 2012. 
30. Kijpornyongpan T, Sereemaspun A and Chanchao C: Dose-dependent cytotoxic effects of menthol on human malignant melanoma A-375 cells: Correlation with TRPM8 transcript expression. Asian Pac J Cancer Prev 15: 1551-1556, 2014.

31. Gkika D and Prevarskaya N: Molecular mechanisms of TRP regulation in tumor growth and metastasis. Biochim Biophys Acta 1793: 953-958, 2009.

32. Sideris M,EminEI,AbdullahZ,Hanrahan J,Stefatou KM,Sevas V, Emin E, Hollingworth T, Odejinmi F, Papagrigoriadis S, et al: The role of KRAS in endometrial cancer: A mini-review. Anticancer Res 39: 533-539, 2019.

33. Liu F, Yang X, Geng $M$ and Huang M: Targeting ERK, an Achilles' Heel of the MAPK pathway, in cancer therapy. Acta Pharm Sin B 8: 552-562, 2018.

34. Sugden PH and Clerk A: Regulation of the ERK subgroup of MAP kinase cascades through $G$ protein-coupled receptors. Cell Signal 9: 337-351, 1997.

35. Krueger JS, Keshamouni VG, Atanaskova N and Reddy KB: Temporal and quantitative regulation of mitogen-activated protein kinase (MAPK) modulates cell motility and invasion. Oncogene 20: 4209-4218, 2001.
36. Samatar AA and Poulikakos PI: Targeting RAS-ERK signalling in cancer: Promises and challenges. Nat Rev Drug Discov 13: 928-942, 2014

37. Cobb MH,Hepler JE, Cheng M and Robbins D: The mitogen-activated protein kinases, ERK1 and ERK2. Semin Cancer Biol 5: 261-268, 1994.

38. Chen WT, Hsu FT, Liu YC, Chen CH, Hsu LC and Lin SS: Fluoxetine induces apoptosis through extrinsic/intrinsic pathways and inhibits ERK/NF-кB-modulated anti-apoptotic and invasive potential in hepatocellular carcinoma cells in vitro. Int J Mol Sci 20: pii: E757, 2019.

39. Mcllwain DR, Berger T and Mak TW: Caspase functions in cell death and disease. Cold Spring Harb Perspect Biol 5: a008656, 2013.

40. Elmore S: Apoptosis: A review of programmed cell death. Toxicol Pathol 35: 495-516, 2007.

41. Wondergem R, Ecay TW, Mahieu F, Owsianik G and Nilius B: $\mathrm{HGF} / \mathrm{SF}$ and menthol increase human glioblastoma cell calcium and migration. Biochem Biophys Res Commun 372: 210-215, 2008.

42. Wondergem R and Bartley JW: Menthol increases human glioblastoma intracellular $\mathrm{Ca}^{2+}$, BK channel activity and cell migration. J Biomed Sci 16: 90, 2009. 